Horizons philosophiques

\title{
L'adaptation de la littérature au cinéma
}

\section{Alain Morency}

Volume 1, numéro 2, printemps 1991

Sémiotiques 2 : théories et champs d'application

URI : https://id.erudit.org/iderudit/800874ar

DOI : https://doi.org/10.7202/800874ar

Aller au sommaire du numéro

Éditeur(s)

Collège Édouard-Montpetit

ISSN

1181-9227 (imprimé)

1920-2954 (numérique)

Découvrir la revue

Citer cet article

Morency, A. (1991). L'adaptation de la littérature au cinéma. Horizons philosophiques, 1(2), 103-123. https://doi.org/10.7202/800874ar d'utilisation que vous pouvez consulter en ligne.

https://apropos.erudit.org/fr/usagers/politique-dutilisation/ 


\section{L’adaptation de la littérature au cinéma}

Depuis ses origines, le cinéma a largement puisé aux sources littéraires, et les adaptations, plus ou moins fidèles, sont le fait de presqu'un film sur deux. Pas toujours réussies ni même transposées de façon intéressante, ces adaptations étonnent. Comment expliquer un tel engouement du septième art pour le littéraire? En fait, la question n'est pas simple et elle en implique bien d'autres auxquelles nous allons tenter de répondre. Dans les réflexions qui vont suivre, nous essaierons donc de préciser les rapports particuliers qui s'établissent entre les différents genres littéraires et le cinéma. II nous faudra traiter des similitudes et des différences entre les deux modes d'expression, toujours dans une perspective d'adaptation, et préciser la nature spécifique de l'un comme de l'autre. Si la littérature et le cinéma se rejoignent et se complètent sous bien des aspects, il faut bien dire aussi que les deux s'opposent jusqu'à un certain point.

\section{Deux expressions}

Un premier questionnement, d'ordre sémiologique, s'impose pour comprendre l'articulation de l'écrit et du filmé. Si on y regarde de près, il semblerait bien que les matériaux signifiants, que la façon d'exprimer et de signifier ne soient pas tout à fait semblables dans les deux lan- 
gages. La littérature s'appuie sur un seul système signifiant, la langue. Cette dernière regroupe des unités de base de premier niveau, discrètes, identifiables, repérables, en un niveau supérieur qui, lui, est porteur de sens. Cette deuxième articulation comprend des unités telles que morphème, mot, syntagme. Une double articulation qui offre des possibilités de construction systématisées et quasi infinies. Or, ce qu'il nous faut noter, en vue d'une comparaison avec le cinéma, c'est le caractère arbitraire, différencié, systématique de la langue. Le mot y est relais de signification, sa nature conceptuelle et «inventée» lui permet de signifier des réalités multiples, tant abstraites que concrètes.

Au cinéma, les choses se passent autrement. Si des unités de base y sont présentes - celles qui correspondraient au premier niveau dans la langue -, elles ne sont guère évidentes ni aisément repérables au cinéma. Tous les éléments signifiants de base de la bande image sont fondus, amalgamés, emportés dans un continuum imageson. Même l'image arrêtée ne facilite pas la tâche du repérage des unités et des liens qui les unissent. L'image est polysémique, ses signifiants sont multiples (ex : cadrage + mouvement de caméra + éclairage + geste + parole...) et simultanés (interaction de ces signifiants entre eux). Bien malin qui pourra isoler un élément de base et expliquer son rôle dans le processus filmique. Serait-ce que le modèle linguistique ne peut rendre compte du fonctionnement particulier de l'image? Sans doute, et c'est davantage au niveau d'une deuxième articulation que la comparaison peut être éclairante. Un plan, une suite de plans, une séquence seraient des équivalents (fort relatifs il faut bien le dire) de la phrase, d'une suite de phrases, d'un épisode. Par le fait même, l'idée d'une adaptation totalement fidèle serait une utopie, alors qu'une transposition de l'écrit au filmé avec des unités plus grandes serait possible. 
Ajoutons à cela que le matériau propre au cinéma se différencie largement de celui de la langue. L'image et le son ne sont guère abstraits, ils semblent plutôt se fondre avec la réalité qu'ils représentent. Avec l'image, peu de distance entre les signes (cette image précisément) et la chose représentée. Alors qu'en littérature (écrite) les mots, les signes ne s'effacent jamais tout à fait, au cinéma signifiant et signifié ont l'air de se confondre. Des signifiants en rapport analogique, en rapport iconique même avec ce qu'ils signifient. Doit-on parler alors de langage d'objet, de signes concrets qui imposent en quelque sorte leur "réalité" de base au langage cinématographique? Pour Jean Mitry : “ll n'est d'image que du concret'". Pour lui, le cinéma n'est pas une langue au sens linguistique du terme : «ll n'y a pas de codification symbolique au cinéma, faute de quoi le film perdrait de son authenticité vivante ${ }^{2}$ ". II nous faut donc constater d'entrée, et cela aura des implications sur la problématique de l'adaptation, la spécificité du code et/ou du langage filmique. L'impression de réalité y est très forte et le spectateur vit cette illusion très agréable d'entrer dans la réalité des êtres et des choses. Christian Metz souligne cette particularité du matériau visuel : "Le cinéma a pour matériau premier un ensemble de fragments du réel, médiatisés par leur duplication mécani$q^{2}{ }^{3}$ ». Nous aurions donc avec la littérature un langage qui devient un monde et au cinéma un monde qui s'articule en langage. Selon Mitry : «Les signifiants filmiques ne sont pas des formes abstraites autour desquelles ou à partir desquelles on pourrait établir certaines lois génératives, mais des faits concrets dont on peut faire qu'ils deviennent... l'expression d'une idée ou d'un sentiment ${ }^{4}$ ». Ce

1. Jean Mitry, Esthétique et psychologie du cinéma, vol. I, p. 127.

2. Ibid., p. 125.

3. Christian Metz, Essais sur la signification au cinéma, vol. I, p. 208.

4. Jean Mitry, La sémiologie en question, p. 117. 
avec quoi nous serons d'accord puisque le cinéma n'a ni langue ni grammaire systématisée, et sa syntagmatique y est bien relative, puisque le syntagme filmique est toujours quelque peu différent d'un film à l'autre. Contrairement à ceux de la langue et de la littérature, les syntagmes du film ne se ramènent pas à un système préexistant aux normes établies et fixes.

Ceci étant dit sur ce qui sépare nos deux expressions, certains aspects les rapprochent et créent une équivalence. La bande image connaît la syntaxe (celle du montage), la composition, le style et rejoint en cela tout à fait l'expression écrite, le littéraire. C'est plus dans le détail que l'adaptation se heurte à des difficultés de transposition d'un langage dans l'autre. Conséquence de sa nature particulière, l'image en montre beaucoup, parfois trop. Alors que l'écrivain peut facilement isoler un élément (notamment grâce à la linéarité du texte), le rendre vague, imprécis, l'abstraire de son contexte, le cinéaste n'aura pas cette souplesse d'entrée en jeu. Un objet ou un personnage à peine évoqués dans le texte deviennent, avec les images, des choses précises avec des caractéristiques visibles, sonores, dans un environnement qu'il n'est pas facile d'abstraire. L'impression de réalité du cinéma fait qu'il faut compter avec des éléments multiples, inhérents à l'image. Or, si cette dernière fascine, si son impact est considérable, sa concrétude toutefois lui rend difficile le concept, les nuances de la pensée, l'abstraction, la fine psychologie. Adapter c'est se rendre compte que, si une image vaut mille mots, transposer ces derniers en images n'est pas chose aisée.

Toujours au chapitre des similitudes et différences, quelques remarques sur les conditions d'écriture et de lecture. L'œuvre littéraire, à l'image de son système signifiant unique, ne requiert qu'un matériel simple dans son écriture. Le film, en contrepartie, s'élabore et se réalise à des niveaux multiples. Auteur, scénariste, caméraman, techni- 
ciens, appareillage lourd et complexe s'y côtoient avec des signifiants qui ont chacun leur spécialiste dans la fabrication de l'image. Un travail d'équipe où l'on ne sait plus toujours où s'arrête le rôle de l'un et où débute celui de l'autre. Cette dimension technique et spectaculaire du film fait qu'il porte, bien davantage que le texte écrit, les marques de son écriture. Cette réalité peut être un handicap lors d'une transposition à l'écran. Les images "trahissent» quelque peu l'époque du tournage, les modes vestimentaires, les progrès technologiques, les tendances filmiques. Par contre, ces images nous laissent des témoignages sans prix sur les plans historique, social, culturel, artistique. Quant aux conditions de lecture, elles sont plutôt différentes que similaires de l'écrit au film. Devant le grand écran, le spectateur n'a guère le choix; il ne peut, comme le lecteur, arrêter le visionnement, suspendre, accélérer ou ralentir le processus. Pas de retour en arrière, le rythme de lecture est lié aux conditions de la bande-image et de sa projection. L'adaptation doit certainement tenir compte de cela sans nécessairement simplifier à outrance la version filmée sous prétexte du déroulement continu et de la lecture soi-disant exigeante du film.

Avant d'aborder les problèmes spécifiques de l'adaptation de certains genres littéraires, examinons une dernière dimension dans la comparaison entre les deux langages. Bien qu'étant un continuum image-son, le film semble plus facile à digérer que l'œuvre écrite, comme si le spectateur y était plus passif. Ce dernier n'aurait pas à imaginer, quelqu'un l'aurait fait à sa place ! En fait, cette passivité n'est qu'apparente. Déjà Mitry disait : "Le film nous délivre du soin d'imaginer ce qu'il nous montre, mais il nous prie de bien vouloir imaginer avec ce qu'il nous montre ${ }^{5}$. Passivité bien relative, puisqu'il s'agirait bien davantage d'une lecture différente de celle du texte. Devant

5. Jean Mitry, Esthétique et psychologie..., vol. I, p. 138. 
le film, tous les sens sont en éveil, l'effort perceptif y est intense, l'imaginaire et l'intellect sont sollicités par les images qui s'impriment en nous. Par des moyens qui lui sont propres, l'expression cinématographique est tout aussi riche et "épuisante", dans le bons sens du terme, que ne l'est l'expression écrite. Reste à savoir jusqu'à quel point ce qui sépare nos deux expressions permet l'adaptation, surtout celle qui se veut fidèle.

\section{Problèmes d'adaptation}

Faut-il s'étonner qu'autant de romans et même de pièces de théâtre soient adaptés à l'écran? Non pas vaguement «inspirés de», mais carrément adaptés, avec ce souci de faire la même chose que ce qu'a fait le texte écrit. De la fidélité, du respect ou de la paresse? II y a de quoi être surpris de telles tentatives, sachant les différences notables entre les deux langages. Tous deux savent fort bien "exprimer", mais les voies qu'ils prennent pour le faire sont forcément différentes. Alors, l'idée même d'adaptation fidèle, comme si les deux langages s'équivalaient et même se confondaient, laisse songeur.

Les cinéastes auraient-ils cette impression, pour ne pas dire cette illusion, qu'on peut transporter le contenu d'une forme de discours à une autre? Sont-ils séduits par la renommée de l'œuvre littéraire, par celle de son auteur, par l'impact sur un large public? Ont-ils le sentiment d'avoir en main une histoire toute prête, déjà écrite, déjà populaire qu'il ne s'agit que de transposer à l'écran? Quiconque est familier avec le langage sait bien que les formes et les structures d'une œuvre sont intimement liées au contenu. Aussi, faire passer une substance d'une forme d'écriture à une autre ne relève pas de la simple traduction mais bien de la réinvention. Traduire un poème, c'est le recréer en quelque sorte. Faire un film à partir d'un roman, c'est également faire autre chose. On dirait, à voir certains films, que les cinéastes n'ont pas suffisamment réfléchi à ce pro- 
blème avec le résultat que le film fait le plus souvent piètre figure face au texte dont il s'inspire.

L'adaptation cinématographique se heurte à plusieurs problèmes. Transposer à l'écran en deux heures de projection une œuvre de cinq cents pages, c'est inévitablement réduire, résumer, dénaturer. On y retrouve le plus souvent les actions, mais on y perd la psychologie, le sentiment de durée, l'atmosphère qui faisaient le charme unique de l'œuvre écrite. Une autre difficulté d'importance : si l'on adapte un roman connu, un grand nombre de spectateurs l'auront lu, s'en seront imprégnés, l'auront imaginé de fond en comble. Devant une adaptation qui se veut fidèle, ils seront confrontés à la vision d'un autre, à des comédiens qui ne recoupent pas nécessairement leur vision des personnages. Le choc risque d'être brutal même si le film a des qualités. De plus, la comparaison est quelque peu faussée dès le départ, une œuvre précédant l'autre. L'inverse est aussi vrai dans ce sens qu'il est bien difficile d'oublier les acteurs et autres éléments d'un film si on lit l'œuvre littéraire après coup. Bien que cette situation soit plus rare que la précédente, elle prouve, en tout cas, l'impact inouï des images du film sur notre imaginaire.

Une solution? Choisir un texte où l'écriture est davantage transparente, où elle joue un rôle mineur, effacé. C'est le cas de récits policiers, d'aventure ou d'espionnage, plus riches en actions qu'en qualités littéraires et donc plus faciles à transposer au cinéma, du moins en principe. On pourrait presque dire que l'idéal, c'est par exemple un mauvais roman dont on ne retiendra que la structure générale, quelques personnages et actions sans se sentir écrasé sous le poids de la richesse littéraire et narrative. lan Fleming au cinéma, c'est plus aisé que Proust. Règle générale, on peut presqu'affirmer que l'adaptation fidèle serait le plus souvent une utopie, voire même une entreprise quelque peu masochiste. II serait sans doute préférable, comme le font de nombreux cinéastes, de s'inspirer d'une 
œuvre tout en la trahissant. Transposer un contenu général, des personnages, des situations, une certaine atmosphère n'est pas impossible. Pour C. Metz : «ll est toujours possible de faire passer dans le film la substance globale du livre, dont néanmoins chacune des pages sera irrémédiablement trahie ${ }^{6}$. Être fidèle, oui, jusqu'à un certain point, tout en faisant forcément autre chose. Orson Welles, Fellini, pour n'en citer que deux, semblent avoir réussi ce pari de rester très près de l'œuvre d'origine, tout en créant quelque chose d'absolument original et personnel. Dans ces cas, et nous aurons l'occasion de le vérifier, le spectateur ne sent pas que le film trahit une œuvre mais bien qu'il s'ajoute à elle dans une nouvelle dimension. Une adaption peut aussi transformer les éléments du texte tout en restant fidèle à son esprit. II n'y a pas de règle stricte, à ce niveau. Qu'elle soit souhaitable ou non, l'adaptation d'une œuvre littéraire au cinéma ajoute une nouvelle dimension, une nouvelle vision et nous permet heureusement de vérifier ce qui rapproche et sépare les deux langages. À ce titre, elle est déjà d'un intérêt considérable.

\section{Les genres littéraires}

Essai, poésie, théâtre et roman vont maintenant occuper notre attention dans les rapports qu'ils entretiennent avec le cinéma. Considérons, en premier lieu, un genre qui n'est pas intimement lié à l'univers filmique, l'essai. Pas ou peu d'adaptation, sauf quelques cas isolés tel Mon oncle d'Amérique de Resnais, et, encore là, ce n'est pas une réelle adaptation, mais un film qui s'inspire des essais de Laborit. Ce genre littéraire évoque la réflexion, l'analyse, l'argumentation, la prise de position. En disant cela, on voit tout de suite que le cinéma s'est davantage dirigé du côté

6. Christian Metz, Essais..., vol. II, p. 102. 
du récit. Toutefois, lorsque le film exprime fortement le point de vue de son auteur, s'il oblige le spectateur à prendre du recul face à la réalité, s'il provoque une prise de conscience, on dira qu'il rejoint l'essai. Le cinéma "d'art et d'essai” s'inscrit un peu dans cette optique; il porte, en tout cas, la marque de son auteur. Moins facile d'accès, ce cinéma demande au spectateur un effort d'interprétation, d'analyse. Texte et film de ce type supposent l'habitude de la réflexion, la familiarité avec l'univers des idées où tout n'est pas donné dès le départ. Le cinéma de Godard, par exemple, se situerait de ce côté, écriture difficile, déconstruction du récit. Un certain cinéma politique, didactique, serait aussi proche parent de l'essai écrit, littéraire ou autre. En fait, ce serait sans doute la télévision, si elle n'était pas devenue ce qu'elle est (et qu'elle va rester), qui pourrait jouer un rôle semblable à celui de la réflexion écrite du type éditorial, chronique, etc. Ce n'est donc qu'indirectement qu'on peut parler d'essai cinématographique. Genre moins facile d'accès, moins séduisant, moins "divertissant", boudé a priori par le large public avide de récits poignants et de belles images.

\section{Poésie}

Avec la poésie, cette forme de littérature qui remonte à la nuit des temps, le cinéma a des liens complexes et intéressants. Pas ou peu question d'adaptation là non plus, puisque, tout comme pour l'essai, on n'y retrouve ni action dramatique ni récit. Pour bien des gens, poésie, univers poétique, se résument à de belles images, à une émotion esthétique. Ce n'est pas tout à fait faux mais c'est un peu comme réduire la poésie à la rime, sa caractéristique la plus apparente. Si la poésie est esthétique dans le texte comme dans l'image, elle est aussi rythme, mesure, musicalité, recherche d'une expression qui cesse d'être transparente, qui ne s'efface pas devant le contenu. Si la poésie est mise en valeur du matériau, harmonie, le cinéma est 
tout cela, avec les éléments signifiants qui sont les siens. Un film, qui ne fait pas qu'être drame ou récit en actions et qui met de l'avant son langage, rejoint en quelque sorte la poésie : jouer avec les mots, les sons, les images, susciter une émotion, toutes choses qui ne s'opposent pas au récit mais s'y greffent et en rendent les signes palpables. "L'expressivité esthétique vient se greffer au cinéma sur une expressivité naturelle7." Esthétique mais aussi éclatement de la signification, connotation forte, tant dans l'image que dans le mot. Au-delà de la dénotation, toujours présente, une connotation qui rend la signification riche, complexe, fuyante. Les mots et les images y rayonnent, deviennent importants en soi : on ne les traverse plus pour aller directement au sens, ils existent pour eux-mêmes. Équivalence plus que similitude, puisque les deux langages ou expressions y prennent des formes différentes.

D'un point de vue rhétorique, on constate une certaine ressemblance quant à deux figures de style majeures. La métaphore poétique, au sens d'un mot pour un autre, surplus de sens, ne se retrouve pas directement au cinéma. Mais, l'autre aspect de la métaphore, confrontation imprévue de deux signifiants, est tout à fait possible et même fréquente dans le film. Étincelle métaphorique, véritable comparaison riche de sens, qu'on retrouve dans les deux langages. L'autre figure, contrepartie de la première, la métonymie, un mot pour un autre, déplacement de la signification, est à la base même de l'expression filmique. Les images ne montrent que partiellement, laissant ce qui n'est pas montré ou entendu, présent implicitement, si l'on peut dire. Ces procédés peuvent être présents dans le film volontairement ou par accident, en tout cas ils y sont, bien que de façon moins omniprésente que dans la poésie écrite.

7. Christian Metz, Essais..., vol. I, p. 81. 
La poésie visuelle est peut-être plus facile à faire partager que celle du texte écrit. Par contre, cette poétique visuelle se résume souvent en clichés, comme s'il était moins ardu et exigeant de faire de la poésie en images qu'en mots. Il faut bien admettre que les films véritablement poétiques, au sens d'une recherche formelle, d'une mise en valeur du langage pour lui-même, ne sont pas légion. Rentabilité oblige, on vise un large public, contrainte moins implacable en poésie écrite. Est-ce là une raison valable? Quoi qu'il en soit, poésie et cinéma entretiennent des liens intéressants même s'ils sont moins évidents que ceux qui relient le théâtre et surtout le roman au monde des images.

\section{Théâtre}

Ce genre littéraire, le seul qui implique la notion de représentation, a beaucoup influencé le cinéma. Et cela ne tient pas uniquement au fait que le cinéma se contentait à l'origine de filmer les pièces, avant de se développer comme langage autonome. Spectacle, mise en scène, dramatisation, personnages, unité de lieu, de temps et d'action allaient fournir au cinéma naissant ses premières composantes. On ne savait pas, à l'époque, que le cinéma allait être autre chose qu'un nouvel art au service d'un autre, plus ancien et plus noble. Si on se contentait, à l'origine, d'enregistrer le drame en plantant la caméra immobile devant la scène, on s'est rendu compte, sous l'impulsion de cinéastes plus créatifs, que le cinéma pouvait être articulé en langage.

Peu à peu, la bande-images s'est libérée du poids que lui imposait l'art de la scène, tout en conservant plusieurs de ses aspects spécifiques. Par le cadrage, les prises de vue et les mouvements de caméra, par le montage, le cinéma s'est constitué en écriture spécifique. Malgré cette évolution irréversible qui l'apparente davantage au récit, au roman, le cinéma est redevable au théâtre de sa concision dramatique, de ses personnages typés, de 
limportance de ses dialogues. Encore qu'au cinéma la parole n'est qu'un élément signifiant parmi tant d'autres, alors qu'à la scène, elle est primordiale. Dans le langage filmique, c'est l'espace qui éclate. II devient celui du monde, comme pour le discours narratif, mais dans sa dimension visible. Espace du film qui est choisi, morcelé, mais infini, ouvert, contrairement à celui de la scène. En effet, même si le spectateur de la pièce est dans le même lieu physique que l'acteur (que la scène soit traditionnelle ou décentrée n'y change rien), l'espace théâtral reste conventionnel, symbolique, stylisé, limité à ses dimensions "réelles". Le spectateur accepte cette convention dès le départ, et il complète par l'imaginaire les éléments manquants, un peu comme le fait le lecteur qui doit imaginer l'univers proposé. C'est un peu comme si le théâtre était à mi-chemin entre un texte qui donne tout à imaginer et une suite d'images qui peut (tout) montrer. Le spectateur du film n'accepte pas de conventions comme cela se fait devant la scène, il attend des images qu'elles se confondent avec la réalité du monde qui l'entoure. Même s'il sait qu'il est au cinéma, le spectateur a une impression de réalité très forte. II est littéralement capté par la bande-images qui se déroule sous ses yeux. C'est un fait que les images du film font parfois plus vrai que ce que nous sommes habitués de voir dans la "Vraie» vie et, ce, grâce à la souplesse et à l'acuité de la vision cinématographique.

À la différence de l'espace scénique, celui du film permet l'ubiquité, le déplacement, le mouvement sous toutes ses formes. Là où le théâtre simule et suggère une ouverture spatiale, le film l'établit dès les premiers instants. Les trois dimensions de l'un offrent paradoxalement moins de possibilités que les deux de l'autre. Le revers de la médaille, c'est que le cinéma est soumis aux conditions du réel. II ne doit pas trop jouer sur le conventionnel, le stylisé, comme l'amplification de l'interprétation ou de la gestuelle qui caractérisent le théâtre. S'il le fait, si par exemple le 
texte fait "théâtral», il perd sa crédibilité, son efficacité. Le spectateur n'est pas dupe, il sent bien de quels moyens dispose le septième art comparé à son vis-à-vis. Le cinéma est d'abord "reproduction fidèle" de la réalité, il faut bien le rappeler, avant même d'être langage. II n'est pas à michemin, comme l'art scénique, entre l'imaginaire et le réel. Une projection de voix excessive, un geste trop souligné s'acceptent et sont même inévitables au théâtre, ce qui n'est pas le cas au cinéma.

Une pièce s'articule autour d'éléments simples, modestes en quelque sorte : un texte, une dramatisation, des personnages, une scénographie. Ce n'est pas par le happening ou la performance ou quelqu'autre tendance à la mode que le théâtre pourra rivaliser avec le monde des images. À chacun ses qualités. Celles du théâtre font qu'il relève quelque peu du sacré, du rite initiatique, de la catharsis. Les acteurs aiment bien y jouer, y sentir cette chimie avec le public, pour revivre soir après soir cette expérience, ce contact, alors qu'au cinéma la représentation est mise en boîte. Nous serons d'accord avec Jean Mitry dans la nuance qu'il apporte sur la nature des deux expressions : "Si donc la présence au cinéma est de l'unité formelle du personnage et du monde, elle est au théâtre, de l'unité formelle de l'acteur et du verbe (...). L'acteur, au théâtre, ne joue pas un rôle, il assume une parole, c'està-dire un personnage tout entier défini par le texte ${ }^{8}$ ". Or, la parole, première à la scène, n'est que seconde dans la bande-images. On verra sans doute de moins en moins de films faits comme des pièces de théâtre. Au delà du problème spécifique de l'adaptation, le cinéma penche plus naturellement du côté du discours narratif. C'est avec le roman qu'il entretient des rapports privilégiés, ce genre littéraire lui aussi ouvert et d'une importance culturelle consi-

8. Jean Mitry, Esthétique..., vol. I, p. 337. 
dérable. Des rapports étroits entre les deux modes d'expression mais aussi des différences qui ne facilitent pas la tâche aux aventuriers de l'adaptation.

\section{Roman}

II n'est pas surprenant de voir autant de cinéastes s'intéresser à la nouvelle et surtout au roman; source privilégiée d'inspiration et pour certains un véritable modèle pour une éventuelle adaptation. Entreprise risquée, dans ce dernier cas, sachant ce que sont les deux langages. Séduisante, par contre, si l'œuvre romanesque est intéressante, riche, facile à adapter. Les cinéastes qui s'appuient sur un bon livre donnent souvent l'impression qu'ils confondent ce qu'ils ont imaginé avec ce qu'ils vont pouvoir filmer. C'est vrai que la parenté est grande entre les deux expressions : roman et film savent raconter, créer un univers, une atmosphère, une psychologie, etc. On sait aussi que le film peut ouvrir son espace à peu près comme le fait le roman. Chose certaine, le cinéma sait montrer. Or, sait-il aussi bien manipuler le récit, a-t-il les mêmes capacités que l'écriture, à ce niveau? Cela reste à voir, aussi allons-nous examiner une première composante du roman, la plus importante peut-être, la narration.

Le théâtre montre, l'écriture raconte et le film joue sur les deux tableaux à la fois. Une même histoire pourrait prendre la forme de l'une comme de l'autre expression. Le récit romanesque, on le sait, passe par les mots et c'est au narrateur qu'échoue le rôle de médiateur du récit. Narrateur personnage ou héros, narrateur omniscient, point de vue interne, externe, ou les deux, la narration écrite offre des possibilités très larges. Par le choix narratif qu'il opère, le romancier oriente son récit, lui donne sa couleur, sa texture. Racontée à la première ou à la troisième personne, I'histoire prend les apparences du fait objectif ou du récit intériorisé, médiatisé par la pensée. Le lecteur sent bien cette présence narrative qui lui permet à la fois de s'iden- 
tifier tout en lui permettant de prendre un peu de recul vis-à-vis ce qui lui est raconté.

Au cinéma, le processus narratif est à la fois semblable et différent. Le film ne fait pas que montrer, que présenter ses personnages et leur drame. II raconte, lui aussi, articule en récit, par ses points de vue multiples, son histoire. Toutefois, la narration ne se présente pas sous les mêmes traits que dans le récit écrit. Le narrateur y est rarement identifié. Quand c'est le cas, narrateur personnage vu et/ou entendu, narrateur extérieur à l'action en voix off, le spectateur ressent fortement cette présence d'une "voix" narrative et cela risque fort d'installer une distance entre le racontant et ce qui est raconté. Le ciné$\mathrm{ma}$, de par sa nature, ne facilite pas cette narration identifiée. L'impression de réalité des images, leur impact, leur concrétude, assure déjà le récit, mais par présentation directe, intense. Pas de relais par les mots qui permet au narrateur de se faire présent et discret à la fois, de se faire un peu oublier, si l'on veut, pour mieux en arriver à ses fins narratives.Tout se passe comme si, dans le film, l'histoire se racontait toute seule, comme si la bande-images assumait la narration sans narrateur identifié. Faut-il parler de "grand imagier", instance ou individu (groupe d'individus puisque le cinéma se fait en équipe) qui serait à la base du récit filmé?

Le film se raconte tout naturellement puisqu'il présente en nous les montrant, les choses racontées. De plus, il montre au présent alors que le récit écrit le fait plus naturellement au passé.

En lieu et place d'un narrateur, le film focalise, par exemple, par (vision subjective) et sur un personnage (vision objective). II choisit des points de vue multiples dans son récit qui équivalent, jusqu'à un certain point, à la narration écrite. Cadrage, jeu de caméra, choix des plans et montage surtout, assurent le récit en douce, comme si l'histoire se narrait toute seule. Le film raconté résulte évidem- 
ment d'une sélection d'éléments, d'une composition et rejoint tout à fait le récit écrit, mais en remplaçant le narrateur par une instance narrative propre au langage cinématographique. Le je, le il, sont remplacés par la présentation focalisée des choses mêmes. II s'agit là d'une nuance de taille entre les deux modes d'expression. Dans un cas, raconter avec des mots inventés pour "représenter' le réel et, dans l'autre, raconter avec des images qui se confondent avec le réel à ce point qu'elles n'ont l'air que de le "présenter». Quand on présente aussi bien les choses, la narration identifiée peut devenir inutile, redondante ! Comment, par contre, assurer ce rôle narratif, considérable dans bien des romans, que permet le récit écrit? Le cinéma semble voué à présenter le réel dans son extériorité, alors que le roman est très à l'aise (grâce aux mots) avec l'intériorité de ses personnages. Le roman permet une durée, une psychologie lente et subtile, le cinéma étale un espace avant d'installer une durée. Le film peut-il en arriver à la même finesse narrative que le roman, compte tenu de sa nature "spectaculaire"? Éliminons tout de suite une possibilité qui semble s'offrir, celle de réintroduire la même narration que dans le roman, en lui donnant une voix dans le film. II suffit d'avoir vu quelques exemples de cela pour se convaincre que ce n'est là qu'une option très occasionnelle, si l'on veut conserver la crédibilité du récit filmique. Bien des cinéastes ne font pas mieux, en éliminant de leur film-adaptation tout ce qui relève de la narration écrite pour simplement la transposer en actionssituations. Comme si cela était suffisant ! Peut-on penser que quelques actions, présentes dans le texte, additionnées à certains dialogues conservés eux aussi, seraient aussi éclairants que ce que la narration écrite, avec ses indications et explications, nous révèle sur les personnages? On peut en douter sérieusement.

Comment parvenir à une narration intéressante au cinéma, qu'il s'agisse ou non d'adaptation? Pour Jean Mitry, 
il faut «jouer avec les rapports entre commentaire, dialiogue et monologue intérieur rapportés à ce que l'image montre, analyse, suggère ${ }^{9}$ ". II s'agirait de créer des liens entre les différents codes présents au cinéma, langage polysémique, où les signifiants sont multiples et reliés entre eux. Si l'on saisit bien leur fonction respective, on pourrait travailler sur ce qui les relie les uns aux autres. La force du cinéma, nous l'avons signalé précédemment, est dans cette polysémie qui peut espérer rejoindre et, parfois même, dépasser les capacités expressives de la langue et de la littérature. Pour Claire Ropars, le film peut se constituer (comme le roman) en durée par un traitement particulier de sa matière première, l'espace : "ll suffit qu'à travers le défilement des lieux se constitue progressivement un espace imaginaire, dont seul le temps apporte à la fois l'approche et l'absence ${ }^{10}$ ". Pour elle, le cinéma n'a pas spontanément de facilité narrative, ce avec lequel nous sommes d'accord, aussi faut-il que le film s'intériorise le plus possible pour rejoindre la richesse romanesque. II faut "l'élaboration progressive d'un espace intérieur qui se substitue à la représentation continue du lieu d'une action ${ }^{11}$ ». Bresson et Resnais, par exemple, auraient réussi assez bien, selon elle, cette transposition du texte littéraire. Sans doute, mais encore faut-il que le film ne devienne pas trop "littéraire", qu'il conserve son langage propre qui est bien celui d'une bande-images.

Faut-il admettre que, si le film montre aussi bien, c'est le récit écrit qui sait toujours le mieux raconter? Cela tient à la nature spécifique de ces deux modes d'expression, et

9. Jean Mitry, La sémiologie en question, coll. 7ième Art, Le Cerf, Paris, 1989, p. 174.

10. Claire Ropars Weilleumieur, De la littérature au cinéma, coll. U 2, Armand Colin, Paris, 1970, p. 39.

11. Ibid., p. 103. 
rien n'empêche qu'ils peuvent se rapprocher l'un de l'autre, sans cesser d'être ce qu'ils sont fondamentalement. Une narration de film jouerait donc sur plusieurs tableaux. Une interprétation nuancée, bien soulignée par la caméra, qui nous révélerait en partie ce que le narrateur du récit écrit précise, des signifiants d'appoint : bruit, sons, musique qui peuvent aider grandement à nuancer le récit. Le jeu des images, la nature des plans, leur longueur, le montage (véritable syntaxe des images), éléments spécifiques au septième art, et qui assument une bonne partie de la narration. Des ellipses visuelles, une déconstruction de l'espace et de la chronologie qui obligent le spectateur à faire des liens, à imaginer ce qui n'est pas montré, peuvent aider à rendre compte de l'intériorité des personnages. Tous ces éléments, bien manipulés, en arrivent à raconter avec presqu'autant de finesse que le récit écrit. Ce dernier a l'énorme avantage de manipuler des mots, instrument privilégié pour raconter. Équivalence, au mieux, pas vraiment similitude.

Adapter un roman d'action n'est pas trop téméraire, car souvent l'écriture et la narration n'y ont pas un poids considérable. Ces récits déjà "visuels" se transportent assez bien dans le monde des images. Par contre, à l'autre extrême, il sera très difficile, voire suicidaire, de transposer à l'écran un roman qui n'est qu'écriture, narration, univers intériorisé. Proust et Joyce au cinéma, ce n'est pas pour le premier cinéaste venu. Le cinéma a souvent l'air d'avoir de gros sabots quand il veut rendre compte des états d'âme, des pensées, des émotions intérieures subtiles. Bien qu'il ait une puissance connotative intéressante, le langage des images n'est pas spontanément à l'aise avec la narration fine et nuancée.

La narration s'appuie sur des techniques, sur des choix qui s'offrent au romancier pour moduler son récit : description, dialogue, indication d'action, etc. Dans un roman, la description permet de situer l'espace, l'environ- 
nement, les lieux, les personnages. L'écriture étant abstraite au départ, l'écrivain doit constituer cet univers visuel pour que le lecteur puisse l'imaginer. Temps d'arrêt dans le récit, la description montre, fait voir. Au cinéma, aussi bien dire que presque tout est de nature descriptive au départ. Toutefois, lorsque le cinéaste s'arrête, insiste sur les êtres et les choses, lorsqu'il prend le temps de nous les montrer, il fait à peu près la même chose que l'écrivain qui décrit.

Ce que le narrateur nous précise des actions de ses personnages devient au cinéma l'action même, rendue visible. Les indications et explications de pensée sont, quant à elles, moins évidentes comme nous l'avons souligné précédemment. N'allant pas de soi, elles supposent de la part du cinéaste, une utilisation subtile des éléments signifiants.

Dialogue et monologue sont essentiels au récit, tant écrit que filmé. Ils l'actualisent, donnant vie aux personnages. Dans le film, contrainte de réalisme oblige, ils doivent faire très vrai, plus encore que dans le roman et bien davantage qu'au théâtre. Aussi, lors d'une adaptation, il n'est pas toujours utile ni nécessaire de conserver les mêmes dialogues que dans le texte écrit. Le traitement en images étant autre que celui du récit écrit, cela fait faussement fidèle et même non-pertinent.

D'autres procédés d'écriture et de narration, résumés d'action, téléscopages, retours en arrière, anticipations, se retrouvent de façon assez équivalente dans le roman comme dans le film. La nuance entre les deux modes d'expression fait que le film risque de trahir davantage ces différents procédés.

Nous en arrrivons aux dernières composantes des deux types de récit : les personnages. Ils sont au cœur de l'histoire puisque c'est par eux et en eux que les choses se passent. Qu'ils provoquent ou subissent l'action, c'est à eux que le lecteur s'identifie, en les imaginant, en les 
faisant siens. Au cinéma, les personnages sont en quelque sorte imposés. Êtres en chair et en os, incarnés de surcroît par des acteurs qui sont forcément autre chose que ce que l'on avait imaginé en lisant. Devant une adaptation d'un roman qu'on avait lu, on a parfois un choc, une surprise, parfois désagréable, à voir ceux et celles qui ont été choisis comme acteurs. Les voir jouer et parler n'est pas toujours conforme à ce que l'on avait imaginé. Pas de star system dans le roman, le lecteur y joue tous les rôles. Une équivalence entre les deux formes d'expression, mais en même temps une différence appréciable qui tient à la nature des deux expressions.

L'intrigue, la structure de l'action peuvent être tout à fait semblables du roman au film. Adaptation ou pas, nombre de cinéastes se rabattent sur ce seul aspect du récit : personnages en actions. Comme si le cinéma se résumait à cela. On reproche à la littérature populaire d'être souvent stéréotypée, sans finesse psychologique. Or, le cinéma et plus encore la télévision, se contentent peut-être trop de cette dimension très facilement visuelle d'un récit. À voir la majorité des adaptations, c'est un peu le sentiment qui s'en dégage. D'une façon générale, toutefois, on peut parler d'équivalence entre intrigue-action du roman au film.

Le récit filmé présente son espace, le récit écrit fait ressentir la durée. L'une comme l'autre expression ont à se constituer dans une dimension qui ne leur est pas naturelle au départ. Quant aux valeurs véhiculées, aux contenus socio-culturels, aux idéologies, roman et film peuvent tout à fait s'équivaloir, du moins en théorie. Reste une dimension, essentielle, qu'on pourrait identifier comme étant le style et qui fonde l'originalité spécifique d'une œuvre. Une façon unique pour l'écrivain ou le cinéaste d'articuler le discours. Qualité d'écriture, atmosphère particulière, élément central et en quelque sorte indicible qui fait la richesse d'une œuvre. C'est souvent ce qu'on reproche à l'adaptation, de n'avoir pas su rendre, transpo- 
ser, l'essentiel du texte. Non pas de le traduire, ce qui est presqu'impossible, mais de le réinventer dans un autre langage. Ce serait peut-être là l'équivalence véritable, la réussite de l'adaptation.

C'est sur ce dernier point que nous conclurons. Le film est bien autre chose qu'un roman : littérature et cinéma sont complémentaires. Si l'on feint d'ignorer les différences entre les deux modes d'expression, on ne voit plus ce qui relève de leur nature spécifique. Dans le cas des adaptations, le danger est présent sur de nombreux plans comme nous l'avons souligné. D'une façon plus globale, la comparaison entre les deux langages nous permet de voir plus clairement ce qui rapproche et sépare les deux expressions. Leur confrontation ne fait pas de vainqueur, tout au plus révèle-t-elle leurs forces respectives. Reste à vérifier si le cinéma va avoir, dans l'avenir, une influence aussi déterminante sur la littérature que celle qu'elle a eue sur lui jusqu'à maintenant. II faudrait aussi s'interroger sur la nature et le fonctionnement des adaptations faites spécifiquement pour la télévision. En fait, notre analyse s'est voulue sommaire devant un champ d'analyse vaste et complexe qui laisse la porte ouverte à une investigation plus élaborée. 